\title{
ESTRUTURA POPULACIONAL DE POMADASYS CORVINAEFORMIS (STEINDACHNER) (TELEOSTEI, HAEMULIDAE) NA BAÍA DE GUARATUBA, PARANÁ, BRASIL
}

\author{
Paulo de Tarso da Cunha Chaves ${ }^{2}$
}

\begin{abstract}
Populacional Structure of Pomadasys CorvinaEFormis (STEINDAChner) (Teleostei, Haemulidae) at Guaratuba Bay, Paraná, Brazil. Reproductive and biometrical analyses were performed on 1629 individuals of $P$. corvinaeformis collected monthly from October 1993 to September 1996, at Guaratuba Bay, Southern Brazilian coast. The results indicate that the species is more abundant in the mangrove area during winter, when the largest individuals were collected, and spring, when the water temperature is lower and the salinity higher than summer or autumn. The relationship of body weight to body length, for grouped sexes, is "InTW= $-12,0456+$ $3,1831 \ln T L "$. The monthly Condition Factor is highest during summer and lowest during winter. It is suggested that $P$. corvinaeformis is present in the mangrove area during a specific etape of its life cycle and does not use this area to maturation and spawn.

KEY WORDS. Pomadasys corvinaeformis, fishes, mangrove, Guaratuba Bay, Brazil
\end{abstract}

A corcoroca, Pomadasys corvinaeformis (Steindachner, 1868), é uma das espécies com maior representatividade na ictiofauna da Baía de Guaratuba, litoral do Paraná. Em arrastos exploratórios de fundo, conforme a época do ano, sua abundância no manguezal somente é equiparada à de alguns outros Perciformes, em particular os das famílias Sciaenidae e Gerreidae. Comum em todo o litoral brasileiro (MENEZES \& FigUeIREDo 1980), P. corvinaeformis também está representada na Baía de Paranaguá, onde, pelo porte normalmente pequeno de seus indivíduos, não é apreciada comercialmente (M.F.M. Corrêa comunicação pessoal). Sua abundância na região de Guaratuba, entretanto, sugere que a população dessa espécie desempenha uma relevante função, seja por se alimentar de componentes do sistema (trata-se de uma espécie onívora - CostA et al. 1995), seja integrando a dieta de peixes e outros animais da comunidade.

Estudos desenvolvidos por CosTA et al. (1995) demonstraram que no litoral do Ceará a população de $P$. corvinaeformis varia sazonalmente em abundância e composição etária. Naquela área, os indivíduos são mais abundantes em meses de elevada precipitação pluviométrica (março a junho), época em que também são mais

1) Contribuição número 963 do Departamento de Zoologia, Universidade Federal do Paraná. Trabalho realizado com auxílios do $\mathrm{CNPq}$ e Funpar.

2) Departamento de Zoologia, Universidade Federal do Paraná. Caixa Postal 19020, 81531-990 Curitiba, Paraná, Brasil. Bolsista do CNPq.

E-mail: poissons@cce.ufpr.br

Revta bras. Zool. 15 (1): 203 - 209, 1998 
freqüentes os indivíduos jovens que os adultos. Comportamento como este demonstra a plasticidade que a estrutura de uma comunidade de peixes pode apresentar, e conhecê-lo em detalhes subsidia planos para a administração dos estoques.

A Baía de Guaratuba, ambiente que sustenta uma contínua atividade pesqueira artesanal e esportiva, é utilizada como área de crescimento e reprodução por várias espécies de peixes (ChAves 1994, 1995; ChAVES \& VENDEL 1997a,b). Da maioria delas, porém, ainda não se conhecem as relações que mantêm com o sistema. A seguir são descritos aspectos da estrutura populacional de $P$. corvinaeformis na área de manguezal, objetivando contribuir com estudos futuros sobre a estrutura da comunidade e a dinâmica das populações de peixes que habitam a região.

\section{MATERIAL E MÉTODOS}

Coletas mensais de Pomadasys corvinaeformis foram realizadas entre outubro de 1993 e setembro de 1996, utilizando rede de arrasto de fundo com portas, na região de manguezal da Baía de Guaratuba (2552'S; $48^{\circ} 39^{\prime} \mathrm{W}$ ) (CHAVES \& VENDEL 1997a). O esforço de pesca foi aproximadamente uniforme, consistindo em cada etapa de um conjunto de três a cinco lances entre quatro e sete minutos de duração, que totalizaram cerca de 20 minutos de arrasto por mês. Em quatro meses (junho, julho, setembro e outubro/94), subamostragem foi realizada a bordo, e apenas parte dos exemplares capturados foi encaminhada para processamento. A profundidade dos locais de coleta variou entre dois e seis metros, e da água de fundo foi realizado um acompanhamento de temperatura e salinidade em 12 meses contínuos (julho/95 a junho/96). Para fins de delimitação sazonal, uma vez que as coletas se realizaram sempre na primeira quinzena de cada mês, considerou-se o verão como compreendido pelos meses de janeiro, fevereiro e março, e assim sucessivamente.

Transportados em gelo para laboratório, 910 do total de 1629 exemplares capturados foram pesados e medidos (comprimento total), e em seguida analisados quanto a sexo e grau de maturação gonadal. Em grande parte das vezes as gônadas não puderam ser reconhecidas, e tal fato apontaria a provável imaturidade do indivíduo. Entretanto, por medida de segurança, esta condição apenas foi assumida quando elas efetivamente foram visualizadas, e neste caso seguiu-se a descrição de VAZZOLER (1996) para teleósteos em geral, considerando-se imaturos os exemplares cujas gônadas eram de dimensões muito pequenas, transparentes, sem granulações, e que apresentavam coloração rósea (ovários) ou branca (testículos). No total, 395 exemplares puderam ser classificados quanto à maturação gonadal.

Os dados biométricos foram tratados de maneira a caracterizar a estrutura da população naquele ambiente, possibilitando sua comparação futura com populações de outras espécies habitantes no mesmo sistema, bem como entre esta população e outras, também de $P$. corvinaeformis, de regiões diferentes. Para tanto, foram calculadas, sempre para sexos grupados: a variação mensal de abundância de captura total, a distribuição mensal de classes de comprimento, a freqüência mensal de ocorrência de indivíduos imaturos em relação a não-imaturos, a relação peso/comprimento dos indivíduos (esta para uma sub-amostra de 632 exemplares, coletados entre outubro/93 e março/96), e a variação dos valores médios mensais do Fator de 
Condição Total, estimado para 611 indivíduos segundo VAZzoLER (1996), conforme segue: $\mathbf{K}=\mathrm{PT} / \mathbf{C T}^{\mathrm{b}}$; sendo: (PT) peso total, (CT) comprimento total, e (b) coeficiente angular da relação peso/comprimento, linearizada mediante utilização do logaritmo dos dados empíricos.
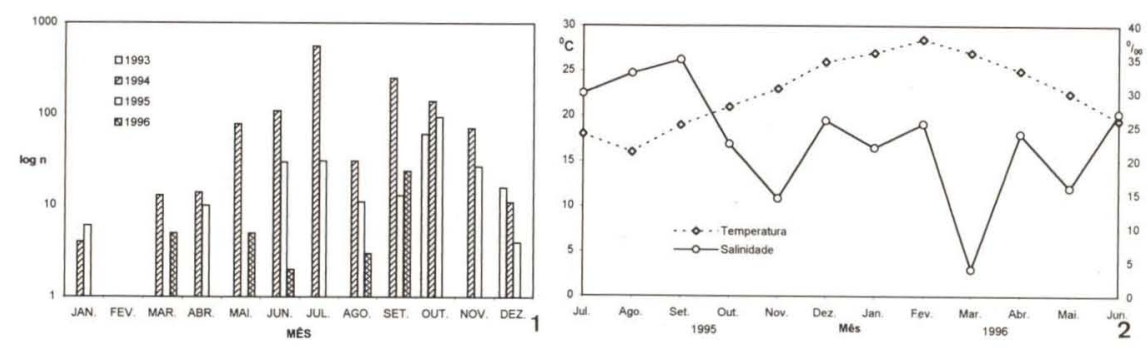

Figs 1-2. (1) Variação mensal da freqüência de ocorrência de $P$. corvinaeformis nas coletas, período outubro/93 a setembro/96. A escala é logarítmica, e o valor 1 corresponde a freqüência zero (exceto em fevereiro/96: 1 indivíduo coletado); (2) valores de temperatura $\left({ }^{\circ} \mathrm{C}\right.$ ) e salinidade (\%o) da água de fundo na região de estudo (médias entre os pontos de amostragem). Período de referência: julho de 1995 a junho de 1996.

\section{RESULTADOS}

A freqüência de ocorrência mensal dos exemplares coletados é apresentada na figura 1. Observa-se que uma maior ocorrência de indivíduos foi registrada em meses a partir do final do outono e até a primavera. Foi também nessa época que a temperatura da água de fundo atingiu seus menores valores, e a salinidade os maiores (Fig. 2). No verão, por outro lado, obtiveram-se as menores abundâncias mensais de captura, época em que a temperatura esteve mais alta e a salinidade mais baixa.

Do total de indivíduos sobre os quais se realizou biometria, o menor media $76 \mathrm{~mm}$ de comprimento total, enquanto o maior $200 \mathrm{~mm}$. A distribuição de comprimento segundo o mês está apresentada na figura $3 \mathrm{a}, \mathrm{b}$ e revela que, enquanto exemplares de menor porte são mais abundantes em meses de inverno, os de maior o são ao final da primavera/início do verão. Nesta época não foram encontrados indivíduos menores que $110 \mathrm{~mm}$.

A análise do grau de maturação dos exemplares cujas gônadas puderam ser reconhecidas revelou que todos os indivíduos examinados nos meses de fevereiro a outubro estavam imaturos (porém, em março nenhuma gônada foi reconhecida para classificação). Indivíduos não-imaturos apenas foram coletados nos meses de novembro, dezembro e janeiro, e em freqüência nunca superior a $50 \%$ (Fig. 4).

A distribuição Peso total (PT)/Comprimento total (CT) resultou numa curva do tipo exponencial (Fig. 5), e a regressão linear obtida através do logaritmo dos dados empíricos foi: $\ln \mathrm{PT}=-12,0456+3,1831 \ln \mathrm{CT}(\mathrm{r}=0,9802)$ 

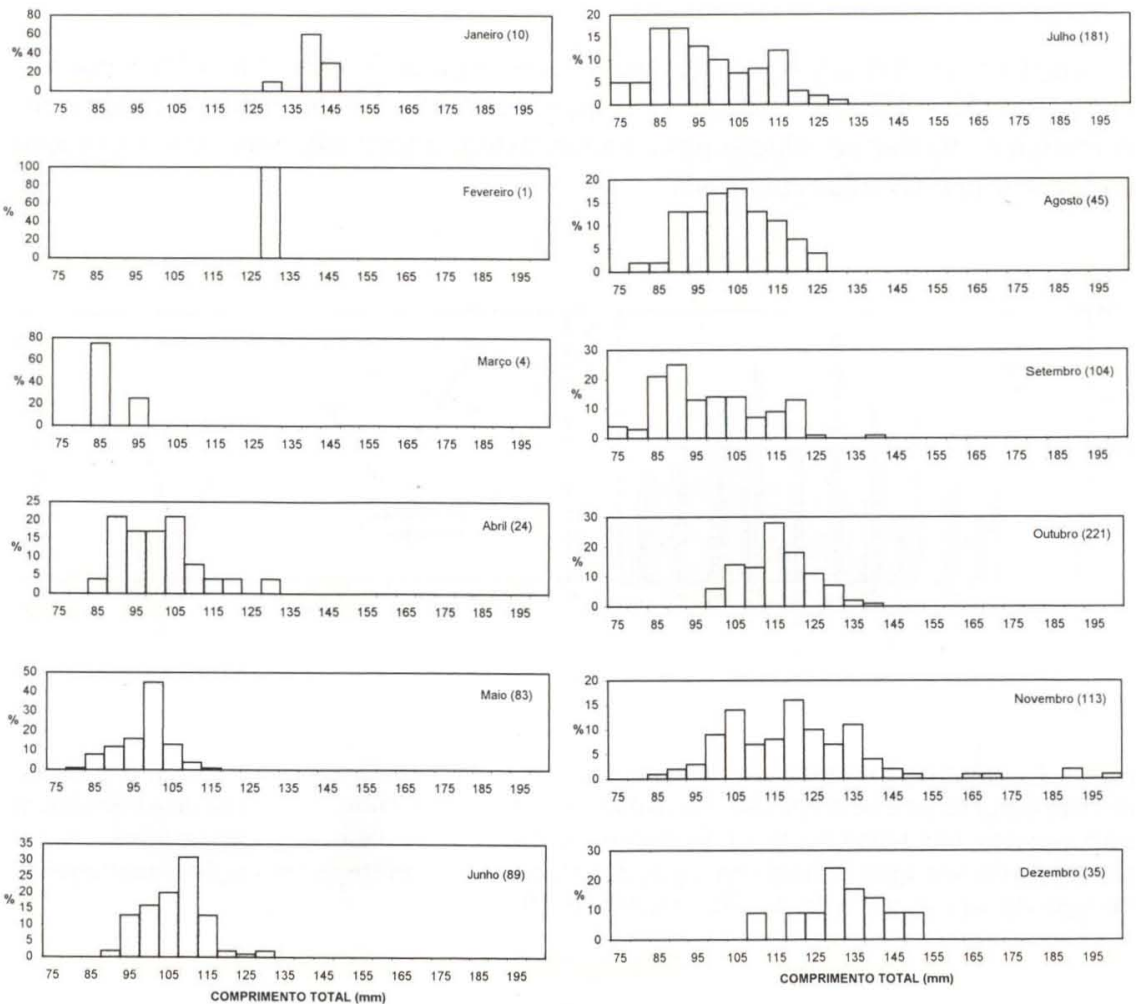

Fig. 3. Distribuição mensal de comprimento dos exemplares de $P$. corvinaeformis capturados no periodo outubro/93 a setembro/96. Entre parênteses, indica-se o número analisado. (a) Janeiro a junho; (b) julho a dezembro.
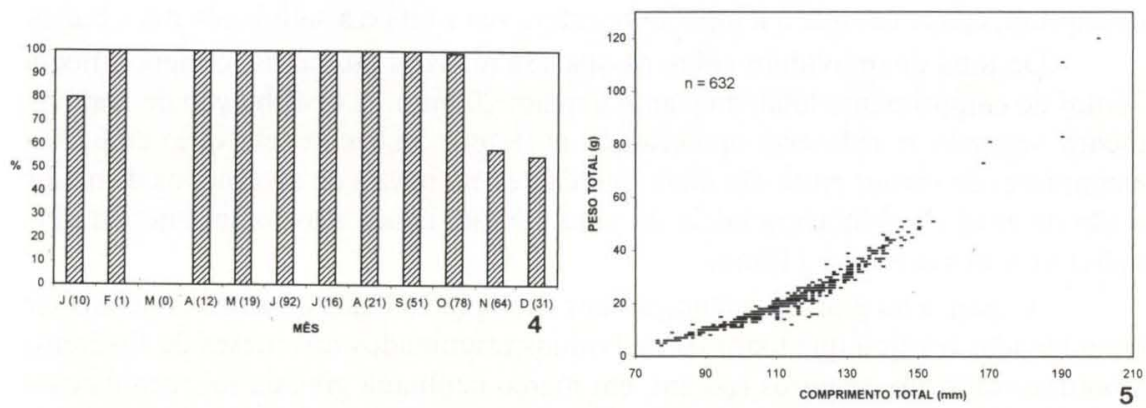

Figs 4-5. (4) Freqüência porcentual mensal de ındividuos imaturos em relação ao número total analisado (entre parênteses), composto por imaturos e não-imaturos; (5) distribuição dos dados empíricos da relação peso/comprimento, obtida a partir de 632 individuos coletados. Período de referência: outubro/93 a setembro/96. 
A variação mensal do Fator de Condição Total, considerando o valor 3,1831 como expoente do denominador, está representada na figura 6. Observa-se que as maiores médias ocorreram no verão, enquanto as menores no inverno.

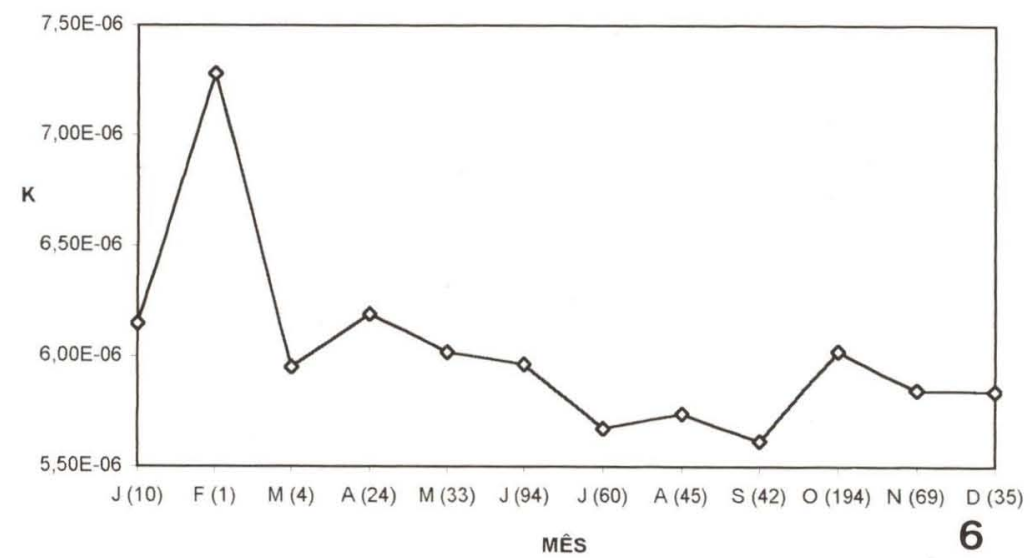

Fig. 6. Variação mensal do Fator de Condição $(K)$ médio, a partir de exemplares coletados entre outubro/93 e setembro/96. Entre parênteses, o número de indivíduos analisados em cada mês.

\section{DISCUSSÃO}

O tamanho máximo normal de Pomadasys corvinaeformis nas regiões Sul e Sudeste do Brasil, segundo Menezes \& Figueiredo (1980), é de $250 \mathrm{~mm}$. No manguezal da Baía de Guaratuba os indivíduos foram capturados com até $200 \mathrm{~mm}$, um comprimento maior que aqueles com que normalmente já se trabalhou biologicamente a espécie em outras regiões - $131 \mathrm{~mm}$ na Baía de Paranaguá, $30 \mathrm{~km}$ ao norte da de Guaratuba (CORRÊA 1987 - comprimento padrão), e $180 \mathrm{~mm}$ em uma praia

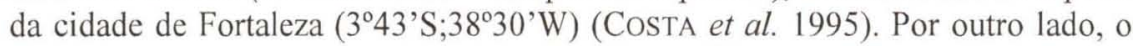
menor comprimento encontrado em Guaratuba $(76 \mathrm{~mm})$ é superior ao mínimo registrado por CORRÊA (1987) em Paranaguá e por CosTA et al. (1995) em Fortaleza, respectivamente $45 \mathrm{~mm}$ (comprimento padrão) e $40 \mathrm{~mm}$. Considerando que o tamanho dos indivíduos de outras espécies comuns nas coletas em Guaratuba dificilmente é inferior a $76 \mathrm{~mm}$, esta diferença não deve indicar inexistência de indivíduos menores na região, mas sim uma limitação imposta pela seletividade da rede de pesca.

$\mathrm{Na}$ faixa de tamanho estudada, Pomadasys corvinaeformis tem sua maior abundância em meses de inverno e primavera, quando a temperatura da água é mais baixa e a salinidade mais elevada, conseqüência de menor precipitação pluviométrica na região. Este comportamento é inverso ao registrado por CoSTA et al. (1995) em Fortaleza. Os autores não citam valores nem de temperatura, nem de salinidade, mas informam que a abundância da espécie - mesmo de indivíduos na faixa de tamanho dos deste trabalho - acompanha de forma direta a intensidade de chuvas. No manguezal de Guaratuba, na época de chuvas (verão, salinidade mais baixa), a 
espécie é menos abundante. A diferença registrada entre as populações das duas regiões pode estar associada às particularidades de cada ambiente fisiográfico estudado (interior de uma baía em Guaratuba, e praia aberta de uma enseada, em Fortaleza), mas também ser um indicativo de que chuvas e salinidade não são fatores isolados a determinar a abundância de $P$. corvinaeformis num ambiente costeiro.

Os dados obtidos referentes a estágio de maturação gonadal evidenciam que o manguezal da Baía de Guaratuba não é um sítio para desova de $P$. corvinaeformis. Em todas as estações do ano foram analisados exemplares com tamanho superior a $125 \mathrm{~mm}$ ( $50 \%$ do tamanho máximo normal para a espécie), mas nem assim indivíduos em atividade reprodutiva foram detectados. Embora a região seja utilizada como local de desova por outros teleósteos (Chaves 1994, 1995; Chaves \& VENDEL 1997a,b), para P. corvinaeformis ela provavelmente apenas atua como área de crescimento. É possível que um deslocamento populacional de saída do manguezal ocorra no início do verão, e outro de regresso no final, visto que nesta estação os indivíduos de todos os tamanhos examinados são pouco comuns na região. A desova provavelmente ocorre durante este período, seja em outra área interna à Baía, seja na região marinha adjacente.

Analisando-se o coeficiente angular da relação peso/comprimento, cujo valor é próximo a 3,0, pode-se inferir que o crescimento de $P$. corvinaeformis é do tipo isométrico, o mais comum para peixes em geral (VAZZOLER 1996). As condições para seu crescimento no manguezal parecem sofrer influência sazonal. A curva de variação do Fator de Condição indica que os indivíduos estão com peso proporcionalmente maior no verão que no inverno, e com valores intermediários para outono e primavera. Uma vez que em $P$. corvinaeformis a variação de peso não pode ser atribuída a alterações no desenvolvimento gonadal, visto que este manteve comportamento praticamente uniforme entre os exemplares analisados, é provável, então, que reflita diferenças relativas a oferta e procura de alimento no ambiente. De fato, registros de campo relativos às coletas de outubro/ $94 \mathrm{e} \mathrm{janeiro/95}$ apontaram grande quantidade de "gordura visceral" nos indivíduos eviscerados. Os dados permitem concluir, portanto, que embora $P$. corvinaeformis não complete a reprodução no manguezal de Guaratuba, este ambiente é utilizado pela população para, em época anterior ao verão, armazenar reservas somáticas a serem utilizadas durante o período de maturação final e desova.

AGRADECIMENTOS. À bióloga Ana Lúcia Vendel, por seu auxílio nos trabalhos de campo e de laboratório, e a dois Revisores anônimos, pela análise do texto e recomendações efetuadas.

\section{REFERÊNCIAS BIBLIOGRÁFICAS}

Chaves, P.T.C. 1994. A incubação de ovos e larvas em Genidens genidens (Valenciennes) (Siluriformes, Ariidae) da Baía de Guaratuba, Paraná, Brasil. Revta bras. Zool. 11 (4): 641-648.

1995. Atividade reprodutiva de Bairdiella ronchus (Cuvier) (Pisces, Sciaenidae) na Baía de Guaratuba, Paraná, Brasil. Revta bras. Zool. 12 (4): 759-766. 
ChA ves, P.T.C. \& A.L. VEndEL. 1997a. Indicadores reprodutivos das espécies de Citharichthys Bleeker (Teleostei, Pleuronectiformes) na Baía de Guaratuba, Paraná, Brasil. Revta bras. Zool. 13 (3): 669-675.

1997b. Reprodução de Stellifer rastrifer (Jordan) (Teleostei, Sciaenidae) na Baía de Guaratuba, Paraná, Brasil. Revta bras. Zool. 14 (1): 81-89.

CostA, P.S.R.; M.A.M. SANTOS; M.F.A. Espínola \& C. MonTEIRO-NeTO. 1995. Biologia e biometria do coró, Pomadasys corvinaeformis (Steindachner) (Teleostei: Pomadasyidae), em Fortaleza, Ceará, Brasil. Arq. Ciên. Mar, Fortaleza, 29 (1-2): 20-27.

Menezes, N.A. \& J.L. Figueiredo. 1980. Manual de Peixes Marinhos do Sudeste do Brasil. IV. Teleostei (3). São Paulo, Museu de Zoologia, Universidade de São Paulo, 96p.

Vazzoler, A.E.A. DE M. 1996. Biologia da Reprodução de Peixes Teleósteos:

Teoria e Prática. Maringá, EDUEM/SBI, 169p.

Recebido em 26.IX.1996; aceito em 13.IV.1998. 